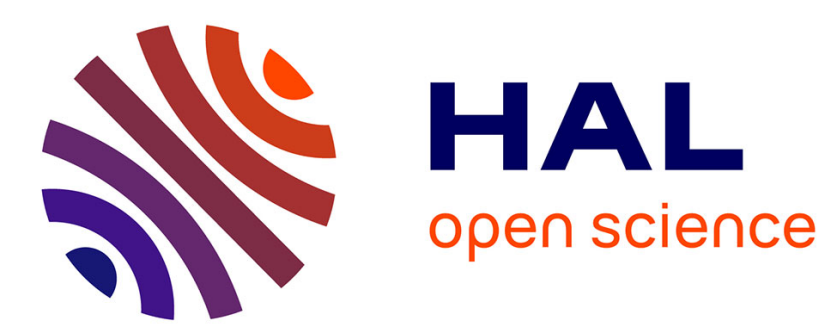

\title{
Enterprise Semantic Modelling for Interoperability
}

Nacer Boudjlida, Hervé Panetto

\section{To cite this version:}

Nacer Boudjlida, Hervé Panetto. Enterprise Semantic Modelling for Interoperability. 12th IEEE Conference on Emerging Technologies and Factory Automation, ETFA'2007, Sep 2007, Patras, Greece. pp.847-854, 10.1109/EFTA.2007.4416868 . hal-00165243

\section{HAL Id: hal-00165243 \\ https://hal.science/hal-00165243}

Submitted on 17 Nov 2007

HAL is a multi-disciplinary open access archive for the deposit and dissemination of scientific research documents, whether they are published or not. The documents may come from teaching and research institutions in France or abroad, or from public or private research centers.
L'archive ouverte pluridisciplinaire HAL, est destinée au dépôt et à la diffusion de documents scientifiques de niveau recherche, publiés ou non, émanant des établissements d'enseignement et de recherche français ou étrangers, des laboratoires publics ou privés. 


\section{Enterprise Semantic Modelling for Interoperability}

\author{
Nacer Boudjlida \\ LORIA - UMR 7503, \\ Nancy-Université, CNRS, INRIA \\ Campus Scientifique, BP 239 \\ 54506 Vandoeuvre CEDEX (F) \\ Nacer.Boudjlida@loria.fr
}

\author{
Hervé Panetto \\ CRAN - UMR 7039, \\ Nancy-Université, CNRS \\ Campus Scientifique, BP 239 \\ 54506 Vandoeuvre CEDEX (F) \\ Herve.Panetto@cran.uhp-nancy.fr
}

\begin{abstract}
The purpose of annotations is to describe the content of "something" and they may be considered as metadata. They are used for a while for text books, articles, hypertext documents and so on. We explore their usage in semantic-based and model-based interoperability, with the aim to make explicit the meaning and the structure of given models (artefacts) to enable not only their understanding, but also their exchange (and their possible transformation) between collaborating actors (human or machine).

We propose categories and types of annotations helpful for expliciting the meaning of models and for easing their exchange within a collaborative context.
\end{abstract}

\section{Introduction}

The purpose of annotations is to describe the content of "something" (we will call the annotated object) and therefore annotations may be considered as meta-data. They are used for a while for text books [16], articles, hypertext documents as well as in some communities like biologists. They may be provided under different forms, like links, paths, notes in and around a text, comments, highlights of important terms and definitions, numbered steps in a process, etc. They may serve various objectives. For instance, the current work on semantic annotations of Web resources and services is intended to serve for sophisticated Web resources retrieval, discovery and composition, as well as for reasoning [6, 8, 9, 13]. In annotated texts [16], annotations can be the basis for information retrieval, for semantic query evaluation and so on.

In our work, we consider annotations for enterprise systems and applications interoperability purposes. We are mainly concerned with enterprise interoperability based on enterprise models [18] which are an abstract representation of some enterprise perspectives. As such, an enterprise model may encompass various types of models (we will call enterprise model perspective), such as an information model, a process model, a resource model, a decision model, etc. Models being artefacts expressed in a given notation (an enterprise modelling language), it is usually understandable to its only designer. Interoperability assumes, at least, that two "actors" can exchange and operate on an "object". Therefore they need to unambiguously interpret the exchanged object. In our work, "objects" are models or part of models enriched with annotations.

We identify a variety of annotations that might be attached to models, together with the variety of their purposes [3, 4]. We distinguish between human readable annotations and machine readable ones, the latter being called semantic annotations. Furthermore, we consider that annotations are produced by an annotation provider and their aim is to render an annotated model understandable and/or processable by "someone else" (the human or the machine annotation consumer) than the model producer. We assume that any part or any fragment (that we call the annotation grain) within an enterprise model perspective may be annotated. We also assume that the annotation grain is identifiable and that multiple annotations can be attached to it. The purpose that is pursued in this work being annotation of enterprise models for interoperability [2], a requirement is that the interoperating actors (the annotation provider and the consumer) agree on the meaning of the variety of annotations that may be attached to an annotation grain (i.e. the semantics of the annotations themselves [1]), as well as on the form under which annotations are actually provided (the annotation scheme).

For this purpose, we define a conceptual annotation scheme, i.e. an information structure dedicated to the actual provision of the annotation content. We also propose an initial typology of annotations (decoration, linking, instance identification, etc.) with their proper meaning. The provided annotations can then serve for: 
1. Improving the models readability ;

2. Making explicit a model designer's decision and assumptions;

3. Enabling the unambiguous exchange of models between collaborating agents (human and/or machines),

4. Enabling the transformation of models from one notation to another one,

5. Enabling the traceability of a model thanks to its annotation by the successive transformations that may be applied to some initial model,

6. Applying formal techniques to analyse enterprise models with regard to formal properties (like soundness and completeness) and to infer further knowledge from the annotated models.

This paper mainly deals with items 1 to 4 .

Another concern of this work is the possible architecture and components of a software platform that supports the annotation of enterprise models. This platform is expected to include, in an integrated manner, facilities or services for the support of enterprise modelling, ontology management and annotations. However, due to space limitations, this topic is not considered in depth here.

The paper is structured as follows. We first define types, and services for annotating enterprise models (section 2). Then, in section 3, we elaborate on what is in an annotation and what are the purposes of annotations for interoperability. Section 3 discusses the required categories of annotations that enable model exchange and transformation. Concluding remarks are in section 5.

\section{Annotations: Definition and Purposes}

We introduce hereafter a typology of annotations, the link between the annotations and the ontologies together with requirements for annotations to be consistently first provided and second interpreted. Then we briefly discuss which kind of support is required to extend current enterprise modelling tools with annotation facilities.

\subsection{Types and Semantics of Annotations}

Different types of annotations may be distinguished; these include:

1. Textual annotations that consist in added notes and comments to the annotated "object".

2. Link annotations that extend the textual annotation notion: the annotation content is reachable through a provided link.

3. Semantic annotations: while textual annotations and link annotations are primarily intended toward humans, semantic annotation content is some semantic information intended to be human readable as well as machine readable and processable.
Further, annotations may appear as informal (like a margin note, while reading a book or an article) or formal: that means that the annotation expressions may range from annotation expressed according to given structural standards (like RDF and RDF Schema) to annotations expressed in some sound and well-founded language (like First Order Logic, Description Logic, etc.). It is clear that the more we are close to the later types of language the more the machine-readability of the annotation could be performed.

This assumes that no implicit assumptions and no ambiguity persist to enable a common interpretation and understanding of the annotations. Therefore, in addition to the annotation definition language that is used, a common understanding of the provided annotations is required. Part of this common understanding may rely on the use of one or several ontologies that provide "a representation of a shared conceptualization of a particular domain" [17]. It means that the conceptualization has to be agreed by the authors of the annotation (who are called the annotation providers) and by the ones who exploit the annotations (the annotation consumers); and that also means that, for some types of annotations, the annotation contents are linked to concepts in the ontology.

Additionally, in order for the annotation to be interpreted and processed consistently, annotation consumers need to understand the meaning of the variety of types of annotations that are provided to them [1]. Indeed, “... meaningless data cannot acquire meaning by being tagged with meaningless metadata..." [14]. Let us consider a very simple example: a theorem being stated in a document, what types of annotations may be associated with it? One can annotate that theorem providing a link to its demonstration or providing the demonstration itself; someone else may annotate the document with a list of possible applications of the theorem, etc. Therefore, the interpretation and the processing of the theorem annotations are then obviously different.

As an illustration of types of annotations, [1] introduces a classification of possible types, considering a resource U\#X annotated with a concept expression C, $\mathrm{U}$ being the URL of a web page and $\mathrm{X}$ being an $\mathrm{XPointer}^{1}$ expression leading to a region of the document:

- Decoration: annotations are comments associated with the resource;

- Linking: annotations are links;

- Instance Identification: the annotated object (U\#X) is an instance of a given class and the annotation content may be a link to that class;

1 XML Framework, W3C, 2003, http://www.w3.org/TR/xptr- 
- Aboutness: no assertion is made about the existence of an instance of the concept $\mathrm{C}$, but there is a loose association with the concept;

- Pertinence: the target of the annotation may be of interest for the annotated object.

The typology of the annotations can further be used by a search engine to classify query answers in accordance with the different types.

\subsection{Services for annotations}

Another matter concerns the way the annotations are effectively provided. Since the content of some types of annotations relies on given ontologies, it seems clear that ontology services (like querying, match-making or browsing an ontology) have to be coupled with annotation services. Moreover, we are progressively moving from manual to automatic or semi-automatic annotation provision. In this framework, few existing annotation platforms use pattern-based and/or machine learning techniques to help in the actual provision of annotations (see [11] for a good survey on this topic and [12] for some freely available annotation platforms). However, to the best of our knowledge, no platform exists for the annotation of models, except $A^{*}$, a tool for ontology-based semantic annotation of business and technical resources (http://leks-pub.iasi.cnr.it/Astar) which works on a RDF representation of models.

From an architecture perspective, [9, 11, 12] distinguish between a proxy based approach and a browser based approach for annotating web resources. In the proxy based approach, annotations and annotated documents are merged by the proxy; the browser only services the merged documents. In a browser based approach, an application of the browser merges the annotations with the documents while browsing. In addition, annotations can be stored separately and provided thanks to an annotation service offered by an annotation server.

These considerations led us to the following scenario (see Figure 1): The annotation provider uses at the same time Ontology management services and annotation management services while modelling the various perspectives that may constitute a model of an enterprise. Annotations that are incorporated into a model or into parts of a model refer to given ontologies. However, as explained in section 4, the single reference to an ontology is not sufficient for the annotation of models.

\section{Annotation Content and Purposes}

A driving question is how and for what purpose a model should be annotated. The variety of purposes of annotations for interoperability has been listed in the section 1. We elaborate, in this section, on the content of annotations.

\subsection{Sources and Targets of Annotations}

Based on the preceding considerations, the following links between an annotated object and the content of the annotation are identified to be useful:

- $\quad$ From the artefact (the model) to a (even informal) definition which expresses the natural meaning of the term used for naming the artefact. The definition may be provided by the available ontologies, by the modelling language as well as by some lexicons or terminology (such as WordNet (http://wordnet.princeton.edu) or Wikipedia (http:/wikipedia.org)). This type of link is considered as a decoration annotation type.

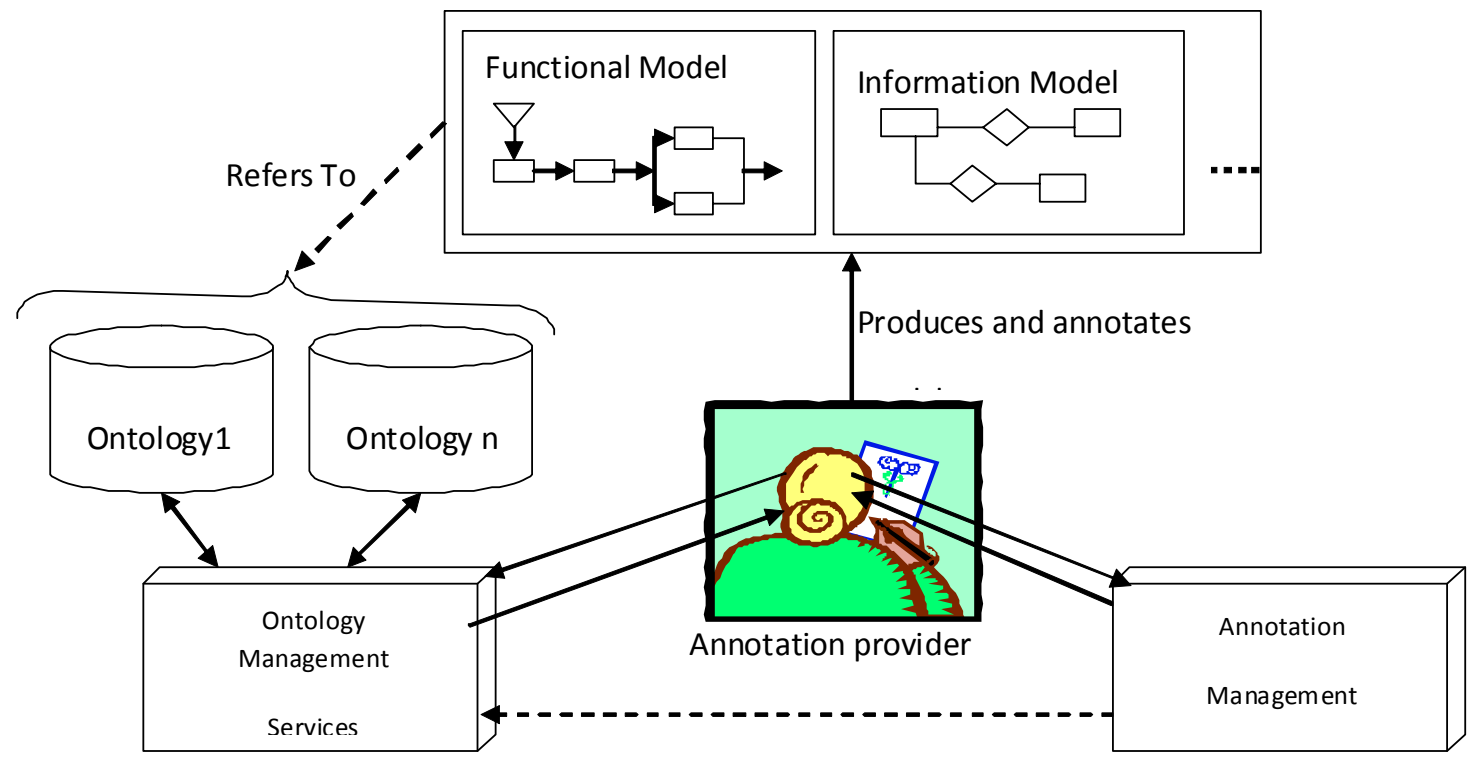

Figure 1. Model Annotation Scenario 
- $\quad$ From the artefact to the application domain (and conversely): in some situations, it is necessary to link a concept in a model to the part(s) of the application domain that concept represents. This type of link might be, for instance, a reference to a piece of text in the description of the application domain, some references to business rules that govern the artefact in the model, etc.

- $\quad$ From the artefact to one or several ontologies and/or to the meta-model of the artefact: this type of link relates a concept in a model to another one in the ontology or in the meta-model of the modelling language (sees section 4).

Another matter of consideration is the granularity of an annotated object: it may range from the whole model (gross grain) to any of its component like an "entity", a relationship, a pre-condition of a process, etc. (fine grain). We assume ${ }^{2}$ that (i) any component (grain) of a model is uniquely identifiable, for instance, thanks to a Uniform Resource Identifier (URI) and (ii) conversely, any reference in the target of a link is also identifiable.

\subsection{From an Annotated Object to its Annotation Target}

The actual establishment of the links, between the annotated object and the corresponding concept in the ontology or in the meta-model, gives rise to the following questions (these questions and remarks typically fall under the domains of model matching and formal specification of the results of the matching [7]):

1. How to automatically or semi-automatically identify and locate the right concepts and/or the relationships in the ontology, starting from the concepts and/or the relationships in the model under annotation? This problem concerns at the same time concepts (usually modelled as classes) as well as relationships between concepts (in the experiments we had, while modelling and annotating, that identification was not made automatically: it has been performed by "visual and manual" browsing of the ontologies and the meta-models).

2. How to actually express the content of the annotation, especially when there is not an exact match between a concept in the model and a concept in the ontology (or in the meta-model)? Indeed, the following situations may arise:

a) There is an exact match between a concept in the model and a concept in the ontology;

b) A concept in the model is more specific than a concept in the ontology;

c) Similarly, a concept in the model is more general than a concept in the ontology;

\footnotetext{
${ }^{2}$ Even though this assumption is not fulfilled by the existing enterprise modelling platforms.
}

d) A concept in the model can be part of a concept found in the ontology (and vice versa);

e) A concept in a model is an instance of a concept found in the ontology (and vice versa)

f) A concept in a model matches (exactly, partly) not a single concept, but possibly more than one concept and relationship (and conversely).

g) No concept in the ontology is satisfactory, as compared to a concept in the model;

Excluding the last item in the preceding list, the other items must be part of the content of the annotation as expressions that use the concepts of the ontology or the ones of the meta-model ${ }^{3}$. The last item requires extending the ontology or the meta-model, adopting another accurate ontology.

\subsection{Annotation scheme}

The preceding considerations, about annotation types, content and so on, conducted to the definition and to the adoption of a common scheme for the provision of the annotations. This scheme includes the following elements:

1. Identification of the annotation;

2. Annotation type (link, aboutness, any newly identified type, etc.);

3. Textual (human readable) description of the annotation content;

4. Identification/location of the target of the annotation: link to an ontology, to a meta-model or to a lexical definition, etc.; this link is assumed to be the URI of its target.

5. Formal definition of the annotation content, when applicable: expression of complementary information, like the type of relationship that holds between the annotated object and the target of the annotation (exact/partial match, more/less general, etc.). The value of this formal definition depends on the types of the annotation. For instance, in an information model perspective, this part may contain the definition of integrity constraints, while, in other perspectives, it may contain the definition of the relevant business rules, etc. Moreover, this part of the annotation scheme is intended to be machine readable and interpretable. Therefore, its content is preferably expressed using a formal language.

For our concern, we defined and used the concrete structured scheme below:

\footnotetext{
${ }^{3}$ In our experiment, UML/OCL has been used for the provision of these types of matching.
} 


\section{$<$ Annotation}

Annotation-Id: Annotation identifier

Informal Content $=$ Natural language comments explaining the intent of the annotation;

Annotation Type $=$ for example, one of:

- Decoration: annotations are comments associated with the annotated object;

- Linking: annotations are links;

- Instance Identification: the annotated object is an instance of a given class and the annotation content (Ref2Ontology) is a link to that class;

- Aboutness: no assertion is made about the existence of an instance of the concept, but there is a loose association with the concept;

- Pertinence: the target of the annotation may be of interest for the annotated object.

Ref2Ontology $=$ references the ontology concept(s) (URI) related to the current model concept

Constraints $=$ might be written using OCL, with references to the ontology or to the meta-model, when these ones are represented as UML diagrams.

One should notice that we deliberately did not make any choice of a concrete syntax for annotations: the preceding scheme may be encoded using XML, or RDF and RDFS, etc. Further, the annotation scheme may be extended with additional elements or attributes.

\section{Model Annotations for Interoperability}

In this section, we discuss the interoperability problem in a heterogeneous context together with the types of annotations that we feel necessary to achieve exchange and transformation of models within that environment. Considering a simple definition of the interoperability as being at the same time connectivity (i.e. the ability of two interoperating actors to exchange "things" like models, information, data, business rules, etc.) and cooperation (i.e. jointly carrying activities to achieve a goal),

\subsection{Annotations in a Heterogeneous environment}

Figure 2 illustrates the variety of heterogeneity that may be encountered between two "actors" that wish to interoperate. Every interoperating actor may use a proper notation for its models (M1 and M2) and consequently, a proper meta-model (MM1 and MM2), a proper ontology (O1 and O2), and possibly, a proper annotation scheme (A1, A2). This is clearly the worst situation where, for connectivity and cooperation, mappings and transformations are required everywhere: between annotation schemes, models, meta-models and ontologies. However, as stated before, a reasonable assumption is that the interoperating actors have a common agreement, at least, on the annotation scheme, on the semantics of the variety of the annotations they use, and they share a common ontology. Besides the already defined classification of annotations (into decoration, linkage, instance identification and so on), we recognize at least three complementary annotations that may be associated with a grain within a model (see Figure 2).

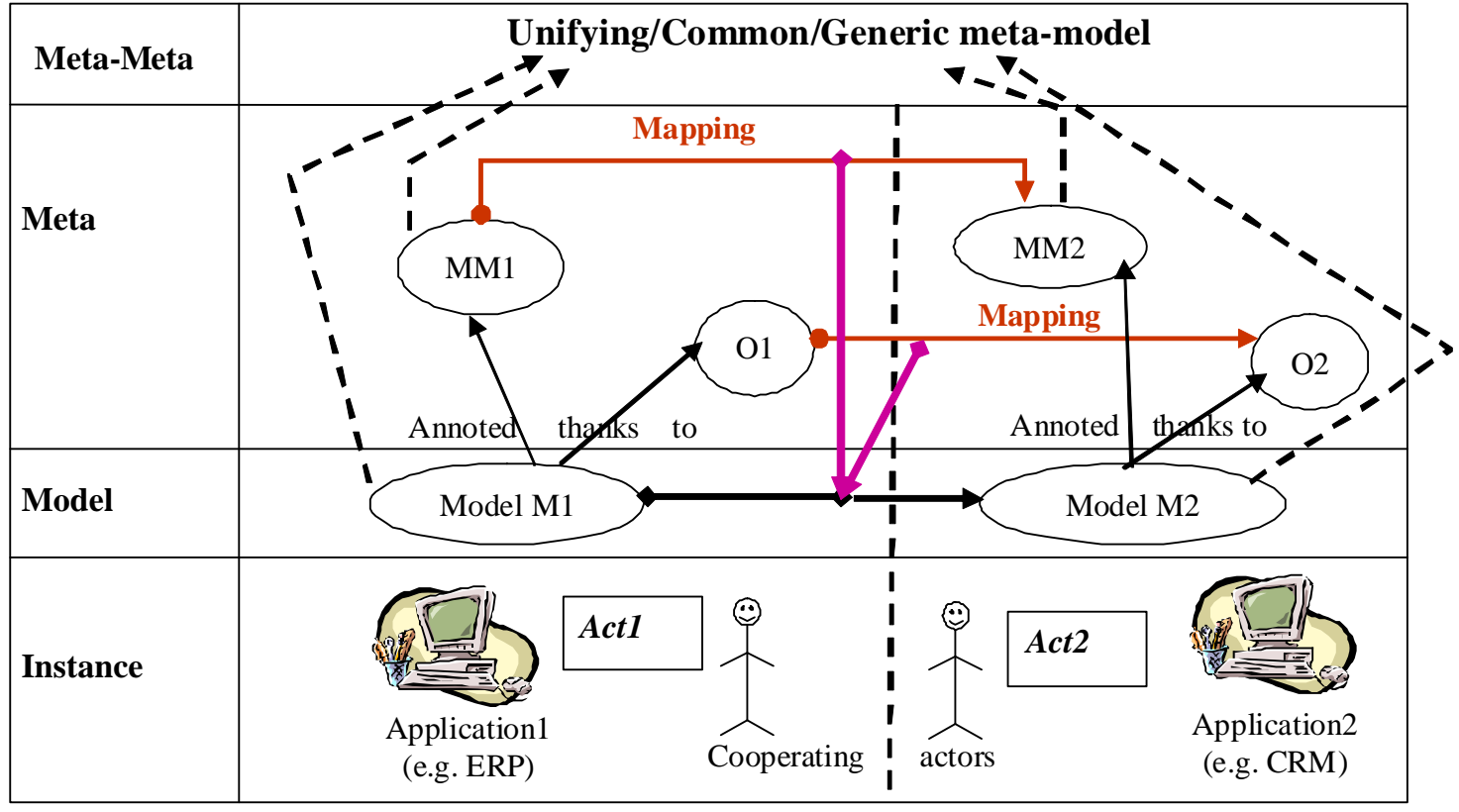

Figure 2. Variety of Models and Annotation 
4.1.1. Structural annotations: they express what a modelling construct has been used to build the annotated grain; they refer to a given meta-model. This metamodel may be a specific meta-model: usually it is the meta-model underlying the notations (or languages) that are used for expressing a model. The meta-model may be a generic one: it is a meta-model that subsumes some given meta-models (for example, the UEML metamodel [5] is a representative of such generic metamodels ${ }^{4}$ ). When a specific meta-model is used, the structural annotations are usually "naturally" inherited from the modelling constructs that are offered by a modelling method. For example, using the Process concept in an enterprise modelling method, an artefact named Proc_P_01 is added to a model: Proc_P_01 is implicitly an instance of Process in the meaning of the method that is used. When a generic meta-model is used in a structural annotation, the interpretation of the annotation by an interoperating actor Act1, requires from Act1 either the knowledge of the generic metamodel, or the availability of mappings between the generic meta-model and the Act1's specific meta-model (this mapping is represented as dotted lines in Figure 2).

Example: Consider an annotated grain $\mathrm{X}$ modelled as a Decision Centre in GRAI and assume that $\mathrm{X}$ has to be sent to a collaborating actor who uses MEGA modelling concepts (MEGA Process, Mega International, http://www.mega.com.). Assume also that UEML 1.0 [5] is used as a generic meta-model. An existing mapping states that a GRAI Decision Centre is more specific than a UEML Activity (i.e. the UEML Activity concept subsumes the GRAI Decision Centre one: GRAIDecision Centre $\subset$ UEML-Activity) and that a MEGA Activity is more general than a UEML Activity (i.e. the UEML Activity concept is subsumed by the MEGA Activity one: UEML-Activity $\subset$ MEGA-Activity). Therefore, at the model level, the grain $\mathrm{X}$ can be unambiguously understood, as a MEGA Activity, by the collaborating partner without any knowledge about the GRAI concepts.

4.1.2. Lexical/Terminological annotations: they express, generally at a "surface semantic level", what the annotated grain represents in the application domain; these annotations concern the names that are associated with the constructed artefacts (example: Proc_P_01) and they usually refer to a commonly agreed definition of terms. The definition may simply be part of a thesaurus or a taxonomy. But, preferably, the definition of terms may come from one or several ontologies, and therefore the annotation content will refer to that ontology(ies). Back to the example: assume that, in the modeller mind, as an intended meaning, Proc_P_01 is a

\footnotetext{
${ }^{4}$ UEML (Unified Enterprise Modelling Language) is a meta-model
} that subsumes some of existing modelling methods. planning activity. The lexical annotation may explicit this meaning thanks to a link between Proc_P_01 and the concept of "Plan Supply Chain" in SCOR, the Supply-Chain Operations Reference-model [10] used here as an ontology.

In addition, ideally, interoperation should respect some principles like:

- The autonomy principle: the consequence of this principle is that an interoperating actor has not to change its way of working, the notations or the vocabulary that the actor is familiar with, the possible naming rules, etc. Lexical notations contribute to the satisfaction of this principle.

- The privacy principle: according to this principle, even in a cooperative process, a partner may hide all or part of its business rules from the partner he/she collaborates with. Therefore, behaviour annotations are especially concerned with this principle and they may be hidden from the annotation consumer.

4.1.3. Behaviour annotations: they are an in-depth expression of the role played by the annotated grain. They can be viewed as the explicit definition of the business logic, the procedures, the rules and the policies that govern the annotated object. The purpose of such a type of annotation is, for example, to ensure that two interoperating processes have the same behaviour (then one can be used instead of the other), or to enforce a defined behaviour from a cooperating process that is sub-contracted, out-sourced or delegated to a collaborating partner. This type of annotation may be provided under various forms (textual descriptions like references to regulations and policies that govern the behaviour of the artefact, enumeration of steps within a process, formal/executable specifications, inter-action diagrams, pre-conditions and post-conditions, rules, etc.) and at various levels of details. When this type of annotations is used for exchanging models between interoperating partners, the level of their details depends on the existing privacy constraints: roughly, the smaller the privacy constraints are, the deeper the level of details should be (this view is quite similar to the "white box"/"black box" metaphor used for software components). For example, the work reported in [10] is a first step toward this aim: it is based on general process ontology and it uses a goal-oriented approach thanks to the annotation of processes or sub-processes with their intended goals (however, the level of details of the annotations do not indicate how the goals can be attained).

Back to the Proc_P_01 example: when receiving the annotated object, an interoperating partner knows its purpose (planning activity) thanks to the lexical 
annotation and, at the enactment level, the behaviour annotations will govern the actual planning activity.

However, if the ontology that is used for expressing the lexical annotations encompasses a commonly agreed or a "standard" behaviour, this type of annotation is redundant and therefore useless.

\subsubsection{Concluding Remarks}

We feel that these types of complementary annotations help the annotation purposes that are concerned in this work: annotation for model exchange, for model transformation and for model traceability. In addition, these purposes are intimately related, since an exchanged model that is notated in a notation $N 1$ may be mapped/transformed into a model expressed in a notation N2. Further, a trace of the model history might be preserved thanks to the expression, as annotations, of the transformation that is applied to the model. Let us elaborate more on model exchange and transformation.

\subsection{Annotations for Model Exchange}

For connectivity purposes, interoperating actors obviously need to exchange a variety of "things". In our context, they are supposed to exchange models or model fragments. In Figure 2, assume that the model M1 is to be sent by the actor Act1 to the cooperating actor Act2.

In a homogeneous context, Act 1 and Act 2 use the same notations for their models as well as the same ontology (in Figure 2, this means that $M M 1$ and $O 1$ are same as $M M 2$ and $O 2$, respectively or, equivalently, that mappings are identity isomorphism). Therefore, fitting M1 with the only lexical annotations (and possibly with behavioural annotations) is sufficient for Act2 to interpret the received model.

In a heterogeneous context, the cooperating actors may use different notations for their models (for example, $M 1$ is notated according to the meta-model MM1 and M2 according to the meta-model MM2) and they may refer to different ontologies (for example, Act1 uses an ontology $\mathrm{O} 1$, while Act2 uses an ontology $\mathrm{O}$, different from 01). According to the autonomy principle, Act2 is not required to have any knowledge about the notations used by Act1. So, what are the types of annotations that have to be attached to a model or a fragment of a model $M 1$ to enable Act2 to unambiguously understand that model or that fragment of model? For the sake of simplicity reasons, we will below use the term model as a synonym for model or fragment of a model.

Now let us consider the variety of situations that can occur in a heterogeneous context:

1. When Act1 and Act2 refer to different ontologies: a mapping must exist (or be discovered) between both the ontologies, and lexical annotations must be attached to $M 1$ to enable Act2 to consistently understand the meaning of $M 1$ as intended by Act 1 , with reference to the ontology used in the annotation.

2. When Act1 and Act2 use different notations to express their models, and therefore different metamodels then, in addition to a mapping between the meta-models MM1 and MM2 that must exist (or be discovered):

a. Structural annotations must be attached to M1 to enable the possible transformation into $M 2$;

b. Lexical annotations must be attached also to M1 to enable Act2 to consistently understand the meaning of $\mathrm{M} 1$ as intended by Act1, with reference to the ontology used in the annotation;

c. Behaviour annotations are not mandatory; if this type of annotation is also attached to M1, it will give Act2 a deeper understanding of what M1 is expected to perform.

3. Similarly, when Act1 and Act2 have an agreement about a common generic meta-model,

a. Mappings between the specific meta-models and the generic one are inherently available;

b. Structural annotations, referring to the generic meta-model, must be attached to the exchanged model;

c. Lexical annotations must also be attached to the exchanged model;

d. Behaviour annotations are not mandatory.

\section{Concluding Remarks}

As concluding remarks, we stress the facts that:

- Annotations have a "syntax", i.e. the form under which the annotation is provided (the annotation scheme), which is understandable by the interoperable actors.

- The annotation "syntax" must be extendible to cover the needs of the variety of purposes, i.e. new properties may be added to the annotation scheme.

- Annotations have a "semantics", i.e. a variety of annotations can be attached to an annotated object and every annotation in that variety has a proper meaning. That meaning must be shared by the interoperable actors. When the annotation consumer is a machine actor, it has to be extended with facilities to interpret (and to possibly react to) the annotations it receives.

- Annotations may serve for different purposes and the variety of annotation types depends from their purpose (their intended usage).

- The provision of a support for annotating models is technically feasible: fragments of technology exist 
today and they mainly need to be made interoperable. And the availability of such a support is clearly an important factor for the scalability of the approach we propose for semantic and model-based interoperability.

However, an important feature is missing: it is the one that permits the automatic or the semi-automatic provision of the annotations given some models. Our current activity deals with the integration of that variety of services to support model annotations together with the exploration of paths toward the support for the annotation provision.

\section{References}

[1] S. Bechhofer, L. Carr, C.A. Goble, S. Kampa and T. MilesBoard. The Semantics of Semantic Annotation. In proceedings of CoopIS/DOA/ODBASE, p. 1152-1167, Irvine, California, USA, October 30 - November 1, 2002, LNCS\# 2519.

[2] N. Boudjlida. "Semantic Annotations for Interoperability". Invited talk at the International Research School “Ontologies, a Smart Way Toward Interoperability”, Paris, France, IAE, 13 April 2006.

[3] N. Boudjlida \& al. A practical experiment on semantic enrichment of enterprise models in a homogeneous environment. INTEROP Deliverable DTG4.1. June 2006.

[4] C. Diamantini and N. Boudjlida. About Semantic Enrichment of Strategic Data Models as Part of Enterprise Models. In J. Eder and S. Dustdar \& al. eds, Proceedings of the 4th Business Process Modelling Workshops, pages 348--359, Vienna, Austria, September 2006, LNCS\# 4103, Springer-Verlag, June 2006, ISBN 10: 3-540-38444-8.

[5] G. Berio, K. Benali, N. Boudjlida and M. Petit. A Unified Enterprise Modelling Language for Enhanced Interoperability of Enterprise Models. In Proceedings of the 11th IFAC Symposium on Information Control Problems in Manufacturing, INCOM'04. Salvador, Brazil, April 2004.

[6] S. Hamri, N. Boudjlida and M. Boufaïda. An approach for Building an OWL Ontology for Workflow Interoperability. Proceedings of the third International Conference on Interoperability of Enterprise Software and Applications IESA'07, Madeira Island, Portugal, 28-30 March 2007, p. 357-364. R.J. Gonçalves, J.P. Müller, K. Mertins and M. Zelm eds, Springer-Verlag.

[7] M. Huza, M. Harzallah and F. Trichet. OntoMas: a Tutoring System Dedicated to Ontology Matching. Proceedings of the third International Conference on Interoperability of Enterprise Software and Applications I-
ESA'07, Madeira Island, Portugal, 28-30 March 2007, p. 377_388. R.J. Gonçalves, J.P. Müller, K. Mertins and M. Zelm eds, Springer-Verlag.

[8] A. Kiryakov, B. Popov, I. Terziev, D. Manov, D. Ognyanoff, Semantic Annotation, Indexing, and Retrieval. Elsevier's Journal of Web Semantics, Vol. 2, Issue (1), 2005.

[9] M-R. Koivunen and R. Swick. Metadata Based Annotation Infrastructure offers Flexibility and Extensibility for Collaborative Applications and Beyond. in Proc. of the KCAP 2001 Workshop on Knowledge Markup \& Semantic Annotation.

[10] Yun Lin, Arne Sølvberg. Goal Annotation of Process Models for Semantic Enrichment of Process Knowledge. In proceedings of the 19th International Conference on Advanced Information Systems Engineering (CAiSE'2007), Springer-Verlag, Trondheim, Norway, June 2007.

[11] L. Reeve and H. Han. Survey of Semantic Annotation Platforms. In Proceddings of the ACM Symposium on Applied Computing, SAC’05, Santa-Fe, New Mexico, USA, March 13-17, 2005.

[12] Semantic Web: Annotation and Authoring Tools. http://annotation.semanticweb.org/tools/

[13] K. Sivashanmugam, K. Verma A. Sheth and J. Miller, Adding Semantic to Web Services Standards, 1rst Intern'l Conference on Web Services (ICWS'03), Las Vegas, Nevada, June 2003, pp. 395-401

[14] John F. Sowa. Ontology, Metadata, and Semiotics. Presented at ICCS'2000 in Darmstadt, Germany, on August 14, 2000. Published in B. Ganter \& G. W. Mineau, eds., Conceptual Structures: Logical, Linguistic, and Computational Issues, Lecture Notes in AI \#1867, Springer-Verlag, Berlin, 2000, pp. 55-81. www.jfsowa.com/ontology/ontometa.htm\#sec1

[15] Supply-Chain Operations Reference-model Version 7, The Supply-Chain Council, 2005, http://www.supplychain.org.

[16] Y. Toussaint. Semantic Annotation of Texts. Invited talk at the $2^{\text {nd }}$ International workshop on Enterprises and Networked Enterprises Interoperability, Vienna, Austria, September 2006. http://www.loria.fr/ nacer/ENEI06/10Invited-Talk.pdf.

[17] M. Uschold and M. Gruninger. Ontologies: Principles Methods and Applications. Knowledge Engineering Review, 11(2), 1996.

[18] F. Vernadat. Enterprise Modeling: Objectives, Constructs \& Ontologies, Tutorial at the 1st EMOI workshop co-located with CAiSE 2004, Riga, Latvia, June 2004. 